\title{
Producción de melón en invernadero: comparación agronómica entre tipos de melón
}

\section{Greenhouse Melon Production: agronomic comparison between types of melon}

\author{
José Eladio Monge-Pérez ${ }^{1}$ \\ Universidad de Costa Rica, Costa Rica
}

\author{
Michelle Loría-Coto ${ }^{2}$ \\ Universidad Estatal a Distancia, Costa Rica
}

\begin{abstract}
Resumen. En esta investigación, se evaluaron siete tipos de melón (Cucumis melo L.): el amarillo, el cantaloupe, el charentais, el galia, el harper, el honey dew y el japonés, producidos bajo invernadero en condiciones hidropónicas en Alajuela, Costa Rica, para determinar su rendimiento y calidad, en los niveles cualitativo (una variable) y cuantitativo (seis variables). Todos los genotipos de los melones tipo amarillo, honey dew y japonés presentaron plantas andromonoicas, mientras que, en los demás tipos, se presentaron genotipos monoicos y andromonoicos. Los datos muestran una amplia variabilidad entre los tipos de melón en cuanto a número de frutos por planta $(1,18-3,26)$; peso promedio del fruto (443,29-837,03 g); rendimiento por planta (685,08-1882,13 g); rendimiento por área (17,79-48,89 ton/ha); firmeza de la pulpa del fruto $(14,91-20,40 \mathrm{~N})$; y porcentaje de sólidos solubles totales $\left(12,48-15,33^{\circ} \mathrm{Bx}\right)$. El mayor número de frutos por planta se obtuvo en los tipos de melón amarillo $(3,26)$ y japonés $(2,79)$; mientras que el menor número, con cantaloupe $(1,48)$ y galia $(1,18)$. Los frutos con mayor peso promedio se consiguieron con los tipos honey dew, cantaloupe y japonés. No se presentaron diferencias significativas entre tratamientos para la firmeza de la pulpa del fruto. El mayor porcentaje de sólidos solubles totales se obtuvo con los melones tipo honey dew, amarillo, harper y japonés. El mayor rendimiento por área se logró con los melones tipo japonés y honey dew (48,89 y 47,51 ton/ha, respectivamente), por lo que se consideran los más recomendables bajo las condiciones en que se desarrolló el ensayo.
\end{abstract}

Palabras clave. Cucumis melo, calidad, rendimiento, ${ }^{\circ}$ Brix, peso del fruto

\begin{abstract}
The Seven melon (Cucumis melo L.) types (Yellow Honey Dew, Cantaloupe, Charentais, Galia, Harper, Honey Dew, and Japanese) grown in greenhouse and hydroponic conditions in Alajuela, Costa Rica, were evaluated to compare their yield and quality both in qualitative (one variable) and quantitative (six variables) terms. All genotypes of Yellow Honey Dew, Honey Dew and Japanese melon showed andromonoecious plants, while the other melon types included both monoecious and andromonoecious genotypes. Data show a wide variability between melon types with respect to number of fruits per plant $(1.18$ - 3.26), mean fruit weight $(443.29$ - 837.03 g), yield per plant (685.08 - 1882.13 g), yield per area (17.79 - 48.89 ton/ha), fruit flesh firmness $(14.91-20.40 \mathrm{~N})$ and percentage of total soluble solids (12.48 $-15.33^{\circ}$ Brix). The highest number of fruits per plant resulted from Yellow Honey Dew (3.26) and Japanese (2.79) melon types, while the lowest results were obtained with Cantaloupe (1.48) and Galia (1.18). Fruits with the highest mean fruit weight resulted from Honey Dew, Cantaloupe and Japanese melon types. There were no significant differences between treatments with respect to fruit flesh firmness. The highest percentage of total soluble solids resulted from Honey Dew, Yellow Honey Dew, Harper and Japanese melon types. The highest yield per area was obtained with Japanese and Honey Dew melon types (48.89 and 47.51 ton/ha, respectively), so these are considered the most recommended under the conditions tested.
\end{abstract}

Keywords. Cucumis melo, quality, yield, ${ }^{\circ}$ Brix, fruit weight

\footnotetext{
${ }^{1}$ José Eladio Monge-Pérez. Ingeniero agrónomo, Investigador de la Estación Experimental Agrícola Fabio Baudrit Moreno, docente de la Sede de Guanacaste, Universidad de Costa Rica. Dirección electrónica: melonescr@yahoo.com.mx

${ }^{2}$ Michelle Loría-Coto. Economista agrícola, Investigadora y docente de la Escuela de Ciencias Exactas y Naturales, Universidad Estatal a Distancia, Costa Rica. Dirección electrónica: michelle loria@yahoo.com
} 


\section{Introducción}

En Costa Rica, los tipos de melón que se producen para exportación son harper, cantaloupe, amarillo, honey dew, galia, piel de sapo, charentais, y orange flesh. Durante el año 2009, 85 \% de las exportaciones a Estados Unidos correspondió a melón tipo harper y cantaloupe, y el $15 \%$ restante a honey dew, mientras que las exportaciones a Europa estuvieron encabezadas por el melón tipo amarillo (58 \%), seguido de harper y cantaloupe (39\%), y galia (3\%) (MongePérez, 2014).

El cultivo protegido de melón presenta varias ventajas sobre el cultivo a campo abierto, como mayor número de cosechas durante el año, precocidad de la cosecha, economía de agua y de fertilizantes, mayor rendimiento y mejor calidad de los frutos (Vargas, Castoldi, Charlo y Braz, 2008). En el cultivo bajo ambiente protegido, se alteran las características ambientales de clima y de suelo: hay menor radiación solar global, evapotranspiración y viento, $\mathrm{y}$ hay mayor radiación difusa, temperatura y humedad relativa del aire ((Martins, Peil, Schwengber, Assis y Mendez, 1998).

Por otra parte, el cultivo de melón en sistema hidropónico permite un control parcial de las condiciones climáticas, menor aplicación de plaguicidas, manejo adecuado del agua y de los nutrientes de acuerdo con el desarrollo del cultivo, la posibilidad de cultivar a mayor densidad y un aumento significativo en la productividad y calidad del producto final (Vargas et al., 2008)

Una ventaja de la producción de melón en invernadero es que se puede cultivar a lo largo del año. En Costa Rica, hay una importante demanda insatisfecha durante la época lluviosa, debido a la muy baja o nula producción (y de baja calidad) de melón a campo abierto, por lo que los consumidores podrían estar dispuestos a pagar precios altos por frutos de alta calidad en esa época; la producción de melón bajo ambiente protegido abriría esta oportunidad comercial para los agricultores (Monge-Pérez, 2016).

Un factor básico para el éxito en la producción hortícola es la selección del genotipo adecuado, pues cada uno de ellos presenta características particulares con respecto al crecimiento de la planta y del fruto. Un genotipo apropiado debe tener características destacadas, a saber: rendimiento alto, resistencia a plagas $y$ enfermedades, calidad del fruto, adaptación a las condiciones ambientales prevalentes en el sitio, aceptación en el mercado y una larga vida poscosecha. La calidad de los frutos de melón está relacionada con características, como la concentración de sólidos solubles totales, la apariencia interna y externa del fruto, el grosor de la pulpa y el sabor, las cuales determinan la aceptabilidad del consumidor. La escogencia de un híbrido de melón sin una evaluación previa puede acarrear perjuicios en la productividad y en la calidad obtenidas (Vargas et al, 2008)

La calidad también está establecida por el tipo de melón, ya que cada uno de ellos muestra diferentes características organolépticas, por ejemplo: color de la cáscara, color de la pulpa, presencia o no de redecilla en la cáscara, aroma, sabor, textura de la pulpa, entre otras (Torres, 1997; Monge-Pérez, datos sin publicar).

El consumo de melón está relacionado con el contenido de sólidos solubles totales, que es responsable del sabor, y con su apariencia externa. El melón es considerado un fruto con poco contenido calórico, bajo en grasas y sodio; no contiene colesterol; y es una buena fuente de potasio, vitamina C (ácido ascórbico) y betacaroteno (provitamina A) (Lester, 1997; Vargas et al, 2008) Además, el melón contiene otros fitoquímicos que favorecen un amplio conjunto de beneficios para la salud; tres de esos compuestos son la betacucurbitacina, el 
litio y el zinc, los cuales parecen jugar un papel en la prevención del cáncer; en el combate de enfermedades como la depresión, la caspa y las úlceras; y en la estimulación del sistema inmunológico (Lester, 1997). En frutos de melón, se presenta mucha variación en el contenido de antioxidantes, tales como el ácido ascórbico y los fenoles, y esta característica depende, principalmente, del genotipo. Los antioxidantes juegan un papel esencial en la salud humana, pues ayudan a prevenir el cáncer, enfermedades cardiovasculares y otras enfermedades crónicas (Lester, 1997; Salandanan, Bunning, Stonaker, Külen, Kendall y Stushnoff, 2009).

En Costa Rica, existe poca investigación sobre la producción en invernadero de los diferentes tipos de melón. El objetivo de esta investigación fue evaluar el rendimiento y la calidad de siete diferentes tipos de melón cultivados bajo ambiente protegido en condiciones hidropónicas, en Alajuela, Costa Rica.

\section{Materiales y métodos}

Para esta investigación se evaluaron 59 genotipos de melón (tabla 1), correspondientes a siete tipos diferentes de melón (Cucumis melo L.): amarillo (5 genotipos); cantaloupe (10 genotipos), charentais (5 genotipos); galia (13 genotipos); harper (18 genotipos); honey dew (5 genotipos); y japonés (3 genotipos). La siembra se realizó en condiciones hidropónicas en el invernadero de hortalizas de la Estación Experimental Agrícola Fabio Baudrit Moreno (EEAFBM), la cual está localizada en Barrio San José de Alajuela, Costa Rica, a una altitud de 883 m s. n. m. Los tipos de melón se caracterizaron segúnla descripción dela literatura (Torres, 1997).

\section{Tabla 1}

Genotipos de melón utilizados en la investigación

\begin{tabular}{|c|c|}
\hline Tipo de melón & Genotipos \\
\hline $\begin{array}{l}\text { Amarillo } \\
(\mathrm{n}=5)\end{array}$ & $\begin{array}{l}\text { Lady (Agriset); Brilliant (Agriset); 16-26-69 (Pandia); Sunshine (Agriset); XY-05-8 (Xin- } \\
\text { jiang Mag-Era) }\end{array}$ \\
\hline $\begin{array}{l}\text { Cantaloupe } \\
(\mathrm{n}=10)\end{array}$ & $\begin{array}{l}\text { UG-505 (United Genetics); UG-4305 (United Genetics); } 325 \text { (Xinjiang Mag-Era); } \\
\text { Charon (DP Seeds); Electra (DP Seeds); Sidewinder Improved (DP Seeds); OTM-10-112 } \\
\text { (Catom); MKS-M220 (Agriset); Holbrook (Hollar); Torreón (United Genetics) }\end{array}$ \\
\hline $\begin{array}{l}\text { Charentais } \\
(\mathrm{n}=5)\end{array}$ & $\begin{array}{l}\text { Gandalf (Nunhems); E-81-8200 (Enza Zaden); Oui (DP Seeds); OT-8-140 (Catom); Skol } \\
\text { (DP Seeds }\end{array}$ \\
\hline $\begin{array}{l}\text { Galia } \\
(\mathrm{n}=13)\end{array}$ & $\begin{array}{l}\text { Solarnet (Nunhems); E-81-2097 (Enza Zaden); 10-26-60 (Pandia); Gala (DP Seeds); Kel- } \\
\text { sey (DP Seeds); Sigal (DP Seeds); Winner (DP Seeds); HSR-4370 (Hollar); OTM-9-21 (Ca- } \\
\text { tom);AX-12-4075 (Agriset);AX-12-4078 (Agriset);HSR-4310 (Hollar);HSR-4402 (Hollar) }\end{array}$ \\
\hline $\begin{array}{l}\text { Harper } \\
(\mathrm{n}=18)\end{array}$ & $\begin{array}{l}\text { M-10 (Xinjiang Mag-Era); Cupid (Agriset); Dainty (Agriset), Red Aroma (Agriset); Red } \\
\text { Lover (Agriset); Crete N.o 1 (Agriset); Infinito (DP Seeds); Sante (DP Seeds); Caribbean } \\
\text { Dream (Rijk Zwaan); WSC-08-46 (DP Seeds); HSR-4366 (Hollar); MKS-M218 (Agriset); } \\
\text { HSR-4406 (Hollar); HSR-4408 (Hollar); HSR-4411 (Hollar); HSR-4413 (Hollar); OTM- } \\
\text { 10-108 (Catom); OTM-10-271 (Catom) }\end{array}$ \\
\hline $\begin{array}{l}\text { Honey dew } \\
(\mathrm{n}=5)\end{array}$ & $\begin{array}{l}\text { T-10 (Xinjiang Mag-Era); Capo Verde (United Genetics); UG-1108 (United Genetics); } \\
\text { Dewlightful (Hollar); Dewluxe (Hollar) }\end{array}$ \\
\hline
\end{tabular}

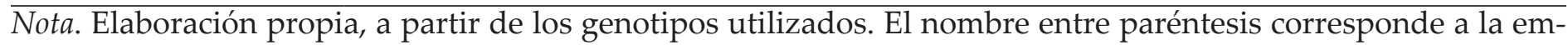
presa productora de la semilla de cada genotipo. 
El ensayo se trasplantó el 3 de noviembre de 2011; las plantas iniciaron cosecha el 9 de enero de 2012, a los 67 días después del trasplante (ddt); y la evaluación de los frutos se realizó hasta el 6 de marzo de 2012 (124 ddt).

El cultivo se llevó a cabo en sacos plásticos rellenos con fibra de coco, de $1 \mathrm{~m}$ de largo, 20 $\mathrm{cm}$ de ancho y $15 \mathrm{~cm}$ de altura. La distancia de siembra fue de 1,54 m entre hileras; y de $25 \mathrm{~cm}$, entre plantas, para una densidad de 2,60 plantas/ $\mathrm{m} 2$. Las plantas fueron sujetadas con dos mallas plásticas, ubicadas a cada lado de las hileras de plantas. Todas las plantas se decapitaron cuando tenían 4 hojas verdaderas (12 ddt) y se dejaron crecer los tallos secundarios libremente. Para cada genotipo, se sembró una parcela con 8 plantas (2 sacos), y todos los datos se obtuvieron a partir de los frutos totales producidos en dicha parcela. Se contó con polinización entomófila en el invernadero, por medio de una colmena de Apis mellifera y otra de Nannotrigona sp.

A partir de los descriptores propuestos para el cultivo de melón (IPGRI, 2003), se seleccionaron las siguientes variables por evaluar:

\section{Variable cualitativa}

Tipo de expresión sexual: se determinó evaluando la presencia de flores hermafroditas (andromonoica) o femeninas (monoica), además delas masculinas, en las plantas de cada genotipo.

\section{Variables cuantitativas}

1. Número de frutos por planta: se obtuvo al registrar el número total de frutos producidos en cada parcela y dividir ese dato entre el número de plantas por parcela.

2. Peso promedio del fruto (g): se midió el peso individual de todos los frutos por cada genotipo y se obtuvo el promedio.

3. Rendimiento por planta (g/planta): se registró el peso de todos los frutos producidos en cada parcela, hasta los $124 \mathrm{ddt}$; la sumatoria de estos datos se dividió entre el número de plantas por parcela.

4. Rendimiento por área (ton/ha): se estimó el peso de los frutos de melón producidos en una hectárea, a partir del rendimiento por planta y de la densidad de siembra.

5. Firmeza de la pulpa del fruto $(\mathrm{N})$ : se midió la firmeza de la pulpa de varios frutos por cada genotipo y se obtuvo el promedio.

6. Porcentaje de sólidos solubles totales $\left({ }^{\circ} \mathrm{Bx}\right)$ : se registró el porcentaje de sólidos solubles totales de varios frutos por cada genotipo y se obtuvo el promedio.

El peso de los frutos se logró/consiguió con una balanza electrónica marca Ocony, modelo TH-IEK, de 5000,0 \pm 0,1 $\mathrm{g}$ de capacidad. El porcentaje de sólidos solubles totales se determinó con un refractómetro manual marca Atago, modelo $\mathrm{N}-1 \mathrm{a}$, con una escala de $0,0-32,0 \pm 0,2^{\circ}$ Brix. Para la evaluación de firmeza del fruto, se utilizó un penetrómetro portátil marca Effegi, modelo FT327 , con una capacidad de 12,5 $\pm 0,1$ kilogramofuerza; y los datos se multiplicaron por un factor de conversión de 9,806 para obtener el dato en Newtons (N); finalmente, se utilizó el puntero cuya base mide $7,5 \mathrm{~mm}$ de ancho.

Se utilizó un diseño experimental irrestricto al azar, donde los tratamientos fueron cada tipo de melón; y cada genotipo correspondió a una repetición. Se realizó un análisis estadístico de varianza y se utilizó la prueba de LSD Fisher con una significancia de $5 \%$ para determinar diferencias entre tratamientos.

\section{Resultados y discusión}

Tipo de expresión sexual

En la tabla 2, se presenta el tipo de expresión sexual de los genotipos evaluados; 39 genotipos fueron andromonoicos $(69,6 \%)$ y 17 monoicos $(30,4 \%)$; esta característica no pudo ser evaluada en el caso de tres genotipos. 
Tabla 2

Tipo de expresión sexual de los genotipos, según tipo de melón

\begin{tabular}{|c|c|c|c|c|c|}
\hline \multirow[t]{3}{*}{ Tipo de melón } & \multicolumn{4}{|c|}{ Tipo de expresión sexual } & \multirow{3}{*}{ Total de genotipo } \\
\hline & \multicolumn{2}{|c|}{ Monoica } & \multicolumn{2}{|c|}{ Andromonoica } & \\
\hline & Total & $\%$ & Total & $\%$ & \\
\hline Amarillo & 0 & 0 & 4 & 100 & 4 \\
\hline Cantaloupe & 3 & 23,1 & 10 & 76,9 & 13 \\
\hline Charentais & 4 & 80,0 & 1 & 20,0 & 5 \\
\hline Galia & 4 & 30,8 & 9 & 69,2 & 13 \\
\hline Harper & 6 & 42,9 & 8 & 57,1 & 14 \\
\hline Honey dew & 0 & 0 & 4 & 100 & 4 \\
\hline Japonés & 0 & 0 & 3 & 100 & 3 \\
\hline Total & 17 & 30,4 & 39 & 69,6 & 56 \\
\hline
\end{tabular}

Nota. Elaboración propia a partir de los datos obtenidos.

Se destaca que $100 \%$ de los genotipos de los melones tipo amarillo, honey dew y japonés mostraron plantas andromonoicas. Esta es una característica importante en cuanto a la producción de frutos por planta, ya que, generalmente, en cultivo en invernadero, se producen más frutos por planta en los genotipos andromonoicos con respecto a los monoicos (J. E. Monge-Pérez, datos sin publicar). Sin embargo, la información sobre esta característica no siempre es fácil de obtener al momento de la compra de la semilla; por lo tanto, es necesario hacer una consulta técnica especializada ante la empresa productora de la semilla o sembrar algunas plantas previamente, para observar sus flores.

Número de frutos por planta

En la figura 1, se presentan los datos del número de frutos producidos por planta, según cada tipo de melón. En este caso, se presentaron diferencias muy importantes entre tipos de melón; los valores encontrados oscilaron entre 1,18 y 3,26 frutos/planta; y los tipos más prolíficos fueron amarillo (3,26 frutos/planta) y japonés (2,79 frutos/planta) (ambos con todos los genotipos andromonoicos), mientras que los menos productivosfueronlosmelonesgaliaycantaloupe.

En el cultivo de melón en invernadero (plantas conducidas en forma vertical), se ha encontrado que normalmente cada planta consigue desarrollar solo entre 2 y 3 frutos, debido a la ocurrencia del aborto natural (Charlo, Castoldi, Vargas y Braz, 2009).

En otras investigaciones sobre producción de melón en invernadero, se han encontrado, para esta variable, los valores que se muestran en la tabla 3 , los cuales indican en general un rango entre 0,30 y 7,30 frutos/planta; los resultados obtenidos en el presente ensayo se ubican dentro de dicho rango. 


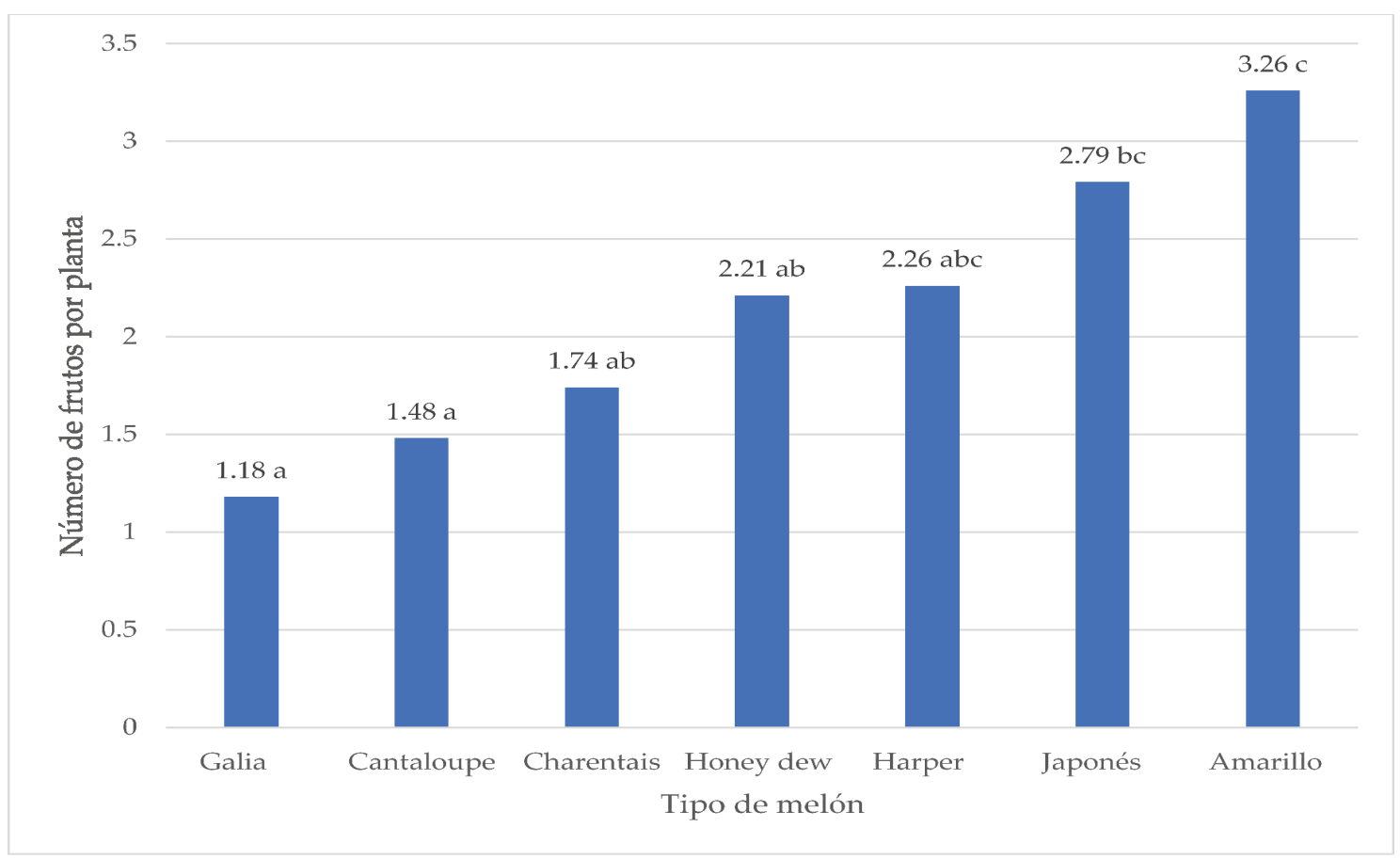

Figura 1. Número de frutos por planta, según el tipo de melón. Nota. Elaboración propia, a partir de los datos obtenidos. Los tratamientos con una letra en común no presentan diferencias significativas entre sí, según la prueba LSD Fisher ( $\mathrm{p} \leq 0,05)$.

\section{Tabla 3}

Número de frutos por planta informado en la literatura para producción de melón en invernadero, según tipo de melón

\begin{tabular}{lll}
\hline Tipo de melón Número de frutos por planta & Refencia
\end{tabular}

\begin{tabular}{|c|c|c|}
\hline \multirow[t]{4}{*}{ Amarillo } & $0,30-1,50$ & Botto, 2011 \\
\hline & 1,55 & $\begin{array}{l}\text { Martins, Peil, Schwengber, Assis y Mendez, } \\
1998\end{array}$ \\
\hline & $2,00-2,58$ & Diaz-Alvarado y Monge-Pérez, 2017 \\
\hline & $2,12-2,76$ & Alvarado-Sánchez y Monge-Pérez, 2015 \\
\hline \multirow[t]{5}{*}{ Cantaloupe } & $0,40-1,10$ & Botto, 2011 \\
\hline & $0,58-1,25$ & Diaz-Alvarado y Monge-Pérez, 2017 \\
\hline & $2,05-3,10$ & Pádua, Braz, Banzatto, Gusmão y Gusmão, 2003 \\
\hline & $3,00-3,90$ & Barni, Barni y Silveira, 2003 \\
\hline & $3,73-4,77$ & Barrientos, 2013 \\
\hline
\end{tabular}




\begin{tabular}{|c|c|c|}
\hline \multirow[t]{2}{*}{ Charentais } & $0,40-1,10$ & Botto, 2011 \\
\hline & 0,93 & Barrientos, 2013 \\
\hline \multirow[t]{9}{*}{ Galia } & $1,30-1,40$ & Botto, 2011 \\
\hline & 1,57 & $\begin{array}{l}\text { Martins, Peil, Schwengber, Assis y Mendez, } \\
1998\end{array}$ \\
\hline & $1,80-5,00$ & haw, Cantliffe y Taylor, 2001 \\
\hline & $2,00-5,00$ & Lamb, Shaw yCantliffe, 2003 \\
\hline & 2,20 & Jani y Hoxha, 2002 \\
\hline & $2,33-2,49$ & Eltez, Tüzel y Boztok, 1999 \\
\hline & $4,18-6,06$ & Abad, Garrido y Fernández, 2006 \\
\hline & $4,50-7,30$ & Rodríguez, Shaw y Cantliffe, 2007 \\
\hline & $4,70-7,20$ & Rodríguez, Cantliffe, Shaw y Karchi, 2006 \\
\hline \multirow[t]{2}{*}{ Harper } & $0,50-0,90$ & Botto, 2011 \\
\hline & 2,10 & Barrientos, 2013 \\
\hline \multirow[t]{2}{*}{ Honey dew } & $0,60-1,00$ & Botto, 2011 \\
\hline & $0,70-1,30$ & Díaz, 2013 \\
\hline \multirow[t]{2}{*}{ Japonés } & $2,00-2,74$ & Costa, Filho, Cavarianni y Barbosa, 2004 \\
\hline & 2,69 & Pádua, Braz, Banzatto, Gusmão y Gusmão, 2003 \\
\hline
\end{tabular}

Nota. Elaboración propia

En relación con los datos reportados por otros investigadores por tipo de melón, los valores obtenidos en el presente trabajo fueron menores para el tipo galia (1,18 frutos/planta, en comparación con un rango entre 1,30 y $7,30)$. Fueron mayores en los charentais $(1,74$ frutos/planta, en contraste con un rango entre 0,40 y 1,10); japonés (2,79 frutos/planta, en comparación a un rango entre 2,00 y 2,74); harper
$(2,26$ frutos/planta, en contraste con un rango entre 0,50 y 2,10$)$; amarillo (3,26 frutos/planta, en comparación a un rango entre 0,30 y 2,76); y honey dew (2,21 frutos/planta, en contraste con un rango entre 0,60 y 1,30). Además, mostraron valores intermedios en el melón cantaloupe $(1,48$ frutos/planta, en comparación a un rango entre $0,40$ y 4,77$)$. 
Peso promedio del fruto

En la figura 2, se presenta el peso promedio del fruto para los diferentes tipos de melón evaluados. Los frutos con mayor peso promedio se encontraron en los melones tipo honey dew $(837,03 \mathrm{~g})$, cantaloupe $(710,04 \mathrm{~g})$ y japonés $(674,03 \mathrm{~g})$, mientras que los valores menores se obtuvieron en los melones amarillo y charentais.

Ciertamente es preferible producir genotipos que presenten frutos con un peso de interés comercial que, en el caso del mercado nacional de Costa Rica, deben ser de más de 600 g (J. E. Monge-Pérez, datos sin publicar); en este caso, los resultados más favorables en el ámbito comercial se hallaron con los melones tipo honey dew, cantaloupe y japonés. En Brasil, se considera que los melones para ser comercializables deben tener un peso entre 800 y 2000 g. (Vargas et a.l, 2008)

En otras evaluaciones de producción de melón en invernadero, se han obtenido para esta variable los valores que se presentan en la tabla 4, que evidencian en forma general un rango entre 161,47 y 2020,00 g; los datos encontrados en el presente trabajo se ubican dentro de dicho rango. Con respecto a los datos informados en otras investigaciones por tipo de melón, los valores obtenidos en el presente ensayo fueron menores en los melones tipo amarillo $(443,29 \mathrm{~g}$, en comparación a un rango entre 511,40 y 2020,00 g); harper $(584,00 \mathrm{~g}$, en contraste con un rango entre 1130,00 y 1300,00 g); honey dew $(837,03$ $\mathrm{g}$, en comparación a un rango entre $972,70 \mathrm{y}$ $2020,00 \mathrm{~g})$; charentais $(479,55 \mathrm{~g}$, en contraste con un reporte de 600,00 g).

Además, se ubicaron en niveles intermedios a los melones galia (596,33 g, en comparación a un rango entre 567,00 y $1800,00 \mathrm{~g}$ ); cantaloupe $(710,04 \mathrm{~g}$, en contraste con un rango entre 161,47 y 1389,30 g); y japonés $(674,03 \mathrm{~g}$, en comparación a un rango entre 578,00 y $1380,00 \mathrm{~g}$ ).

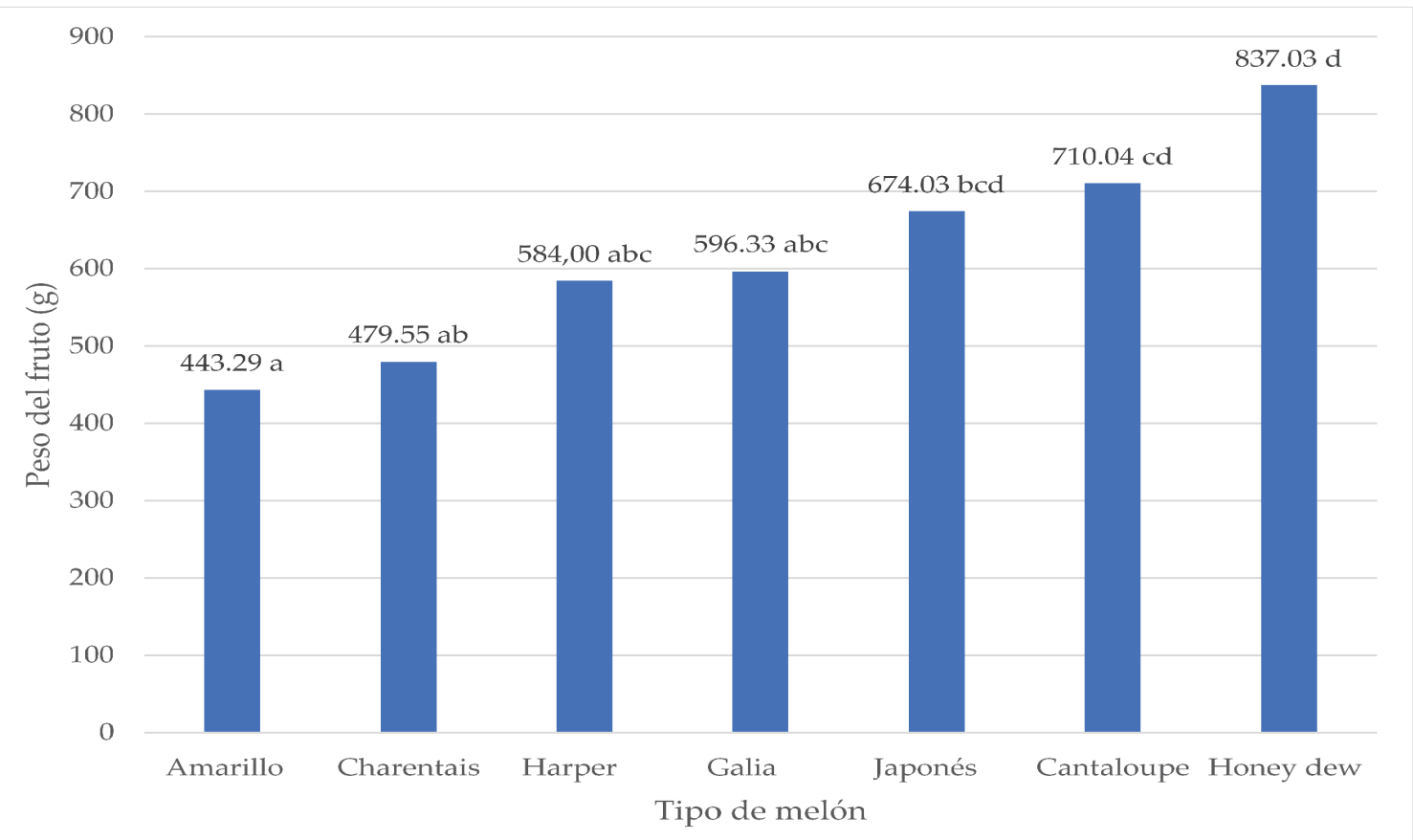

Figura 2. Peso promedio del fruto (g), según tipo de melón.

Nota. Elaboración propia, a partir de los datos obtenidos. Los tratamientos con una letra en común no presentan diferencias significativas entre sí, según la prueba LSD Fisher $(p \leq 0,05)$. 
Producción de melón en invernadero: comparación agronómica entre tipos de melón

Tabla 4

Peso promedio del fruto informado en la literatura para producción de melón en invernadero, según tipo de melón

\begin{tabular}{|c|c|c|}
\hline Tipo de melón & Peso promedio del fruto $(\mathrm{g})$ & Referencia \\
\hline \multirow[t]{4}{*}{ Amarillo } & $511,40-533,00$ & Diaz-Alvarado y Monge-Pérez, 2017 \\
\hline & $732,44-801,76$ & Alvarado-Sánchez y Monge-Pérez, 2015 \\
\hline & 990,00 & $\begin{array}{l}\text { Martins, Peil, Schwengber, Assis y Mendez, } \\
1998\end{array}$ \\
\hline & 2020,00 & Paduan, Campos y Clemente, 2007 \\
\hline \multirow[t]{8}{*}{ Cantaloupe } & $161,47-751,87$ & Morales, 2009 \\
\hline & 362,82 & Rizzo y Braz, 2001 \\
\hline & $530,20-651,90$ & Diaz-Alvarado y Monge-Pérez, 2017 \\
\hline & $695,50-998,30$ & Queiroga, Puiatti, Fontes y Cecon, 2008 \\
\hline & $700,00-880,00$ & Barrientos, 2013 \\
\hline & $754,00-1025,00$ & Barni, Barni y Silveira, 2003 \\
\hline & $791,30-980,30$ & Queiroga, Puiatti, Fontes y Cecon, 2008 \\
\hline & $814,80-1389,30$ & Queiroga, Puiatti, Fontes y Cecon, 2008 \\
\hline Charentais & 600,00 & Barrientos, 2013 \\
\hline \multirow[t]{10}{*}{ Galia } & $567,00-1190,70$ & Lamb, Shaw y Cantliffe, 2003 \\
\hline & $570,00-1550,00$ & Shaw, Cantliffe y Taylor, 2001 \\
\hline & $671,80-950,70$ & Abad, Garrido y Fernández, 2006 \\
\hline & $1000,00-1800,00$ & Rodríguez, Shaw y Cantliffe, 2007 \\
\hline & 1132,00 & Jani y Hoxha, 2002 \\
\hline & 1200,00 & Bezerra, Nunes, Freitas y Silva, 2009 \\
\hline & 1220,00 & $\begin{array}{l}\text { Martins, Peil, Schwengber, Assis y Mendez, } \\
1998\end{array}$ \\
\hline & 1430,00 & Paduan, Campos y Clemente, 2007 \\
\hline & $1430,00-1596,00$ & Eltez, Tüzel y Boztok, 1999 \\
\hline & $1600,00-1700,00$ & Rodríguez, Cantliffe, Shaw y Karchi, 2006 \\
\hline
\end{tabular}

continúa... 


\begin{tabular}{|c|c|c|}
\hline \multirow[t]{2}{*}{ Harper } & 1130,00 & Barrientos, 2013 \\
\hline & 1300,00 & $\begin{array}{l}\text { Mitchell, Cantliffe, Klee, Sargent, Stoffella y } \\
\text { Tieman, } 2008\end{array}$ \\
\hline \multirow[t]{2}{*}{ Honey dew } & $972,70-1129,50$ & Díaz, 2013 \\
\hline & 2020,00 & Bezerra, Nunes, Freitas y Silva, 2009 \\
\hline \multirow[t]{8}{*}{ Japónes } & $578,00-730,00$ & Purquerio, Filho y Barbosa, 2003 \\
\hline & $580,70-669,08$ & Costa, Filho, Cavarianni y Barbosa, 2004 \\
\hline & 693,25 & Rizzo y Braz, 2001 \\
\hline & $715,00-890,90$ & Gualberto, Resende y Losasso, 2001 \\
\hline & $880,00-1220,00$ & Vargas, Castoldi, Charlo y Braz, 2008 \\
\hline & $1080,00-1310,00$ & Charlo, Castoldi Vargas y Braz, 2009 \\
\hline & $1090,00-1260,00$ & Charlo, Galatti, Braz y Barbosa, 2011 \\
\hline & $1160,00-1380,00$ & Charlo, Castoldi Vargas y Braz, 2009 \\
\hline
\end{tabular}

Nota. Elaboración propia.

\section{Rendimiento por planta}

En la figura 3, se presentan los datos de rendimiento por planta para los tipos de melón evaluados. Los mayores valores correspondieron a los melones tipo japonés (1882,13 g/planta) y honey dew (1829,03 g/planta), mientras que la menor producción se obtuvo con los melones galia y charentais.

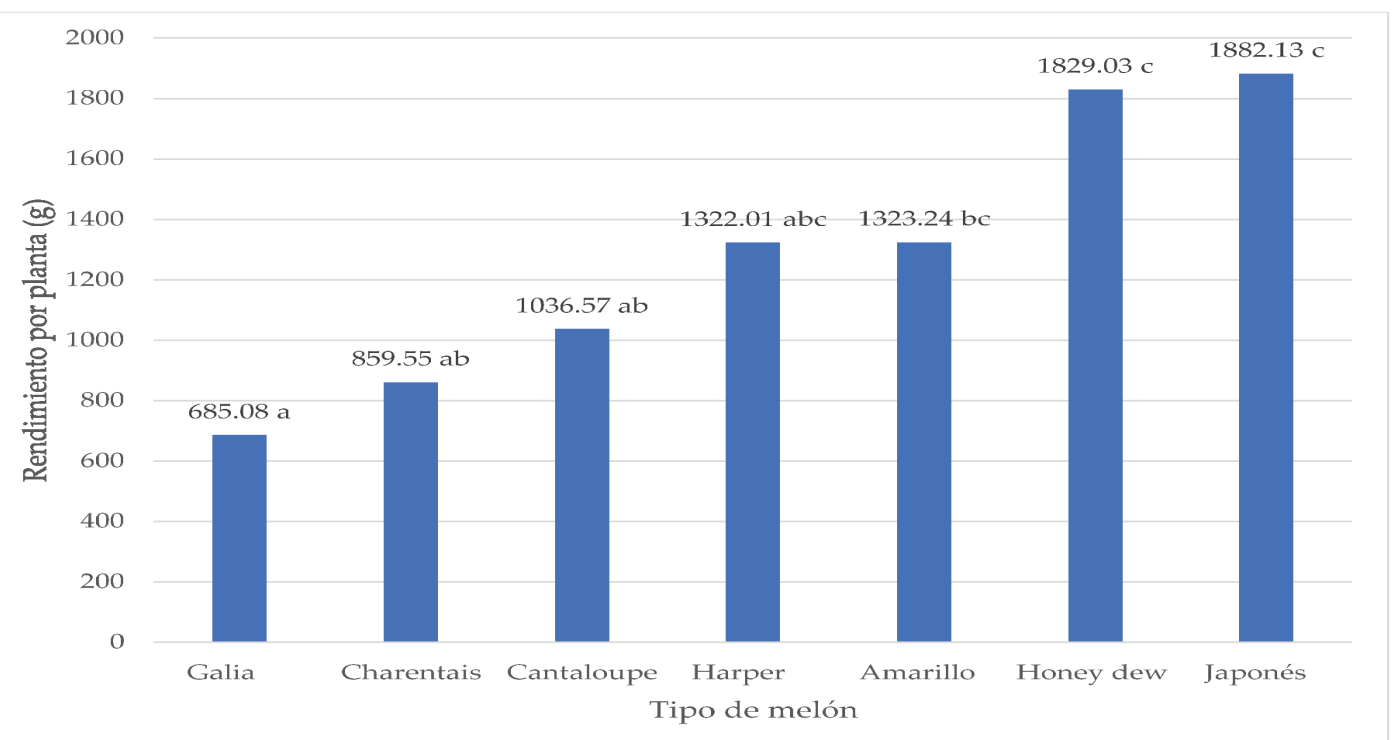

Figura 3. Rendimiento por planta (g), según tipo de melón.

Nota. Elaboración propia, a partir de los datos obtenidos. Los tratamientos con una letra en común no presentan diferencias significativas entre sí, según la prueba LSD Fisher $(\mathrm{p} \leq 0,05)$. 
Entre las razones que explican estos resultados, se debe considerar que los melones tipo japonés y honey dew produjeron más de 2,00 frutos por planta, y sus frutos alcanzaron más de $600 \mathrm{~g}$ de peso promedio, mientras que los melones galia y charentais obtuvieron valores menores en ambas variables. En el caso de los melones cantaloupe, harper y amarillo, solamente alcanzaron valores mayores a dichos niveles para una de esas variables (y no para ambas), lo que explica los valores intermedios obtenidos en el rendimiento por planta.

En otros ensayos de producción de melón en invernadero, se han encontrado, para esta variable, los valores que se muestran en la tabla 5; de manera general, el rango se ubica entre 187,02 y 12000,00 g/planta, por lo que los datos obtenidos en el presente trabajo se ubican dentro de dicho rango. En comparación con los datos reportados por otros investigadores por tipo de melón, los valores obtenidos en el presente ensayo fueron menores para los melones tipo galia $(685,08 \mathrm{~g} /$ planta, en comparación a un rango entre 1000,00 y 12000,00); amarillo (1323,24 $\mathrm{g} /$ planta, en contraste con un rango entre 1480,00 y 1990,00); y harper (1322,01 g/planta, en comparación a un reporte de 1870,00). Para el melón charentais $(859,55 \mathrm{~g} /$ planta, en contraste con un reporte de 660,00) fueron mayores; y presentaron valores intermedios, en el caso de los melones cantaloupe $(1036,57 \mathrm{~g} /$ planta, en comparación a un rango entre 187,02 y 3690,00) y japonés $(1882,13 \mathrm{~g} / \mathrm{planta}$, en contraste con un rango entre 1343,62 y 3230,00). No se encontró información en la literatura para el melón tipo honey dew.

\section{Tabla 5}

Rendimiento por planta informado en la literatura para producción de melón en invernadero, según tipo de melón.

Tipo de melón Rendimiento por planta (g/planta) Referencia

\begin{tabular}{lcl}
\hline Amarillo & $1480,00-1990,00$ & Alvarado-Sánchez y Monge-Pérez, 2015 \\
& 1550,00 & Martins, Peil, Schwengber, Assis y Mendez, \\
& 1998 \\
Cantaloupe & Morales, 2009 \\
& Barni, Barni y Silveira, 2003 \\
& $2730,00-3690,00$ & Barrientos, 2013 \\
& $3260,00-3450,00$ & Barrientos, 2013 \\
Charentais & 660,00 & Shaw, Cantliffe y Taylor, 2001 \\
Galia & $1000,00-4800,00$ & Martins, Peil, Schwengber, Assis y Mendez, \\
& 1990,00 & 1998 \\
& Abad, Garrido y Fernández, 2006 \\
\hline
\end{tabular}


$7700,00-12000,00$

Harper

Japonés
1870,00

$1343,62-2322,81$

$1670,00-2642,00$

$2420,00-2760,00$

$2670,00-3230,00$
Rodríguez, Cantliffe, Shaw y Karchi, 2006

Barrientos, 2013

Costa, Filho, Cavarianni y Barbosa, 2004

Purquerio, Filho y Barbosa, 2003

Charlo, Castoldi Vargas y Braz, 2009

Charlo, Castoldi Vargas y Braz, 2009

\section{Nota. Elaboración propia.}

\section{Rendimiento por área}

En la figura 4, se presentan los datos de rendimiento por área para los tipos de melón evaluados; los valores se ubicaron en un rango entre 17,79 y 48,89 ton/ha. Estas diferencias en rendimiento son muy importantes y se deben tomar en cuenta antes de iniciar un proyecto de producción de melón en invernadero, pues lo deseable es escoger un tipo de melón y un genotipo que produzca un alto rendimiento, aunque también hay que tomar en cuenta la

\section{0}

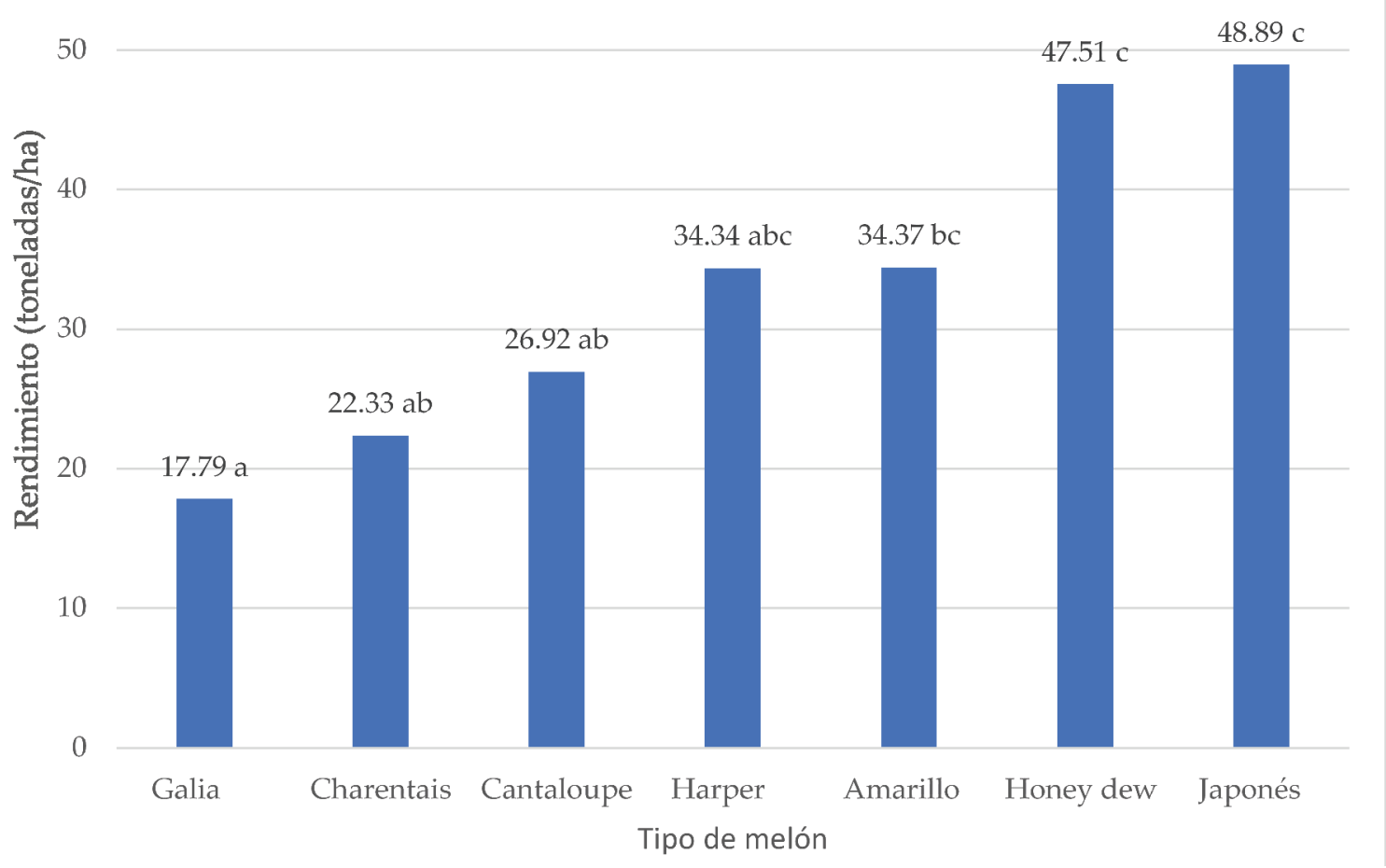

Figura 4. Rendimiento por área (ton/ha), según tipo de melón.

Nota. Elaboración propia, a partir de los datos obtenidos. Tratamientos con una letra en común no presentan diferencias significativas entre sí, según la prueba LSD Fisher $(\mathrm{p} \leq 0,05)$. 
calidad del fruto, que debe ser satisfactoria para el mercado. Los mayores valores se obtuvieron con los melones tipo japonés $(48,89$ ton/ha) y honey dew $(47,51$ ton/ha) y los menores valores se hallaron con los melones galia y charentais.

En otras evaluaciones de producción de melón en invernadero, se han encontrado para esta variable los valores que se presentan en la tabla 6 , que representa de forma general un rango entre 2,45 y 443,00 ton/ha; los datos hallados en el presente ensayo se ubican dentro de dicho rango. En relación con los datos informados en otras investigaciones por tipo de melón, en el presente trabajo, se obtuvieron valores mayores para los melones tipo charentais $(22,33$ ton/ha, en comparación con un rango entre 2,45 y 11,00 ) y harper $(34,34$ ton/ha, en contraste con un rango entre 4,15 y 31,10). Los valores intermedios, para los melones galia $(17,79$ ton/ha, en comparación a un rango entre 9,47 y 443,00$)$; honey dew $(47,51$ ton/ha, en contraste con un rango entre 4,69 y $49,71)$; japonés (48,89 ton/ha, en comparación con un rango entre 30,30 y 131,50); amarillo $(34,37$ ton/ha, en contraste con un rango entre $2,57$ y 51,80$) ;$ y cantaloupe $(26,92$ ton/ha, en comparación con un rango entre 2,71 y 93,90).

Tabla 6

Rendimiento por área informado en la literatura para producción de melón en invernadero, según tipo de melón

Tipo de melón Rendimiento por área (ton/ha)

\begin{tabular}{lcl}
\hline Amarillo & $2,57-11,00$ & Botto, 2011 \\
$15,10-27,50$ & Diaz-Alvarado y Monge-Pérez, 2017 \\
$38,46-51,80$ & Alvarado-Sánchez y Monge-Pérez, 2015 \\
51,80 & Martins, Peil, Schwengber, Assis y Mendez, 1998 \\
Cantaloupe & $2,71-8,12$ & Botto, 2011 \\
& $9,32-41,76$ & Morales, 2009 \\
$10,40-27,60$ & Diaz-Alvarado y Monge-Pérez, 2017 \\
$17,86-28,27$ & Queiroga, Puiatti, Fontes y Cecon, 2008 \\
$23,20-27,30$ & Pádua, Braz, Banzatto, Gusmão y Gusmão, 2003 \\
$36,30-53,16$ & Queiroga, Puiatti, Fontes y Cecon, 2008 \\
$54,30-57,50$ & Barrientos, 2013 \\
$73,30-93,90$ & Barni, Barni y Silveira, 2003 \\
83,20 & Rizzo y Braz, 2001 \\
Charentais & Botto, 2011 \\
& 11,00 & Barrientos, 2013 \\
\hline
\end{tabular}




\begin{tabular}{lcl}
\hline Galia & $9,47-10,42$ & Botto, 2011 \\
41,30 & Jani y Hoxha, 2002 \\
$57,00-70,90$ & Abad, Garrido y Fernández, 2006 \\
66,40 & Martins, Peil, Schwengber, Assis y Mendez, 1998 \\
79,95 & Bezerra, Nunes, Freitas y Silva, 2009 \\
$94,50-98,50$ & Eltez, Tüzel y Boztok, 1999 \\
110,00-443,00 & Rodríguez, Shaw y Cantliffe, 2007 \\
Harper & Rodríguez, Cantliffe, Shaw y Karchi, 2006 \\
& $4,15-6,50$ & Botto, 2011 \\
Honey dew & Barrientos, 2013 \\
$4,69-8,80$ & Botto, 2011 \\
$22,00-31,60$ & Díaz, 2013 \\
49,71 & Bezerra, Nunes, Freitas y Silva, 2009 \\
Japonés & Pádua, Braz, Banzatto, Gusmão y Gusmão, 2003 & Charlo, Galatti, Braz y Barbosa, 2011 \\
& $40,50-45,50$ & Costa, Filho, Cavarianni y Barbosa, 2004 \\
$44,79-77,43$ & Charlo, Castoldi, Vargas y Braz, 2009 \\
$46,40-55,20$ & Gualberto, Resende y Losasso, 2001 \\
$53,14-74,43$ & Purquerio, Filho y Barbosa, 2003 \\
$55,67-88,07$ & Rizzo y Braz, 2001 \\
131,50 &
\end{tabular}

Nota. Elaboración propia.

Como se puede apreciar, hay mucha variación en el rendimiento obtenido según los diferentes investigadores, debido, entre otros factores, al efecto de las condiciones climáticas; al manejo del cultivo (tipo de poda, densidad de siembra, riego, fertilización, sustrato utilizado, manejo fitosanitario, etc.); al tipo de melón utilizado; y al genotipo evaluado.

Firmeza de la pulpa del fruto

En la figura 5, se presentan los datos de firmeza del fruto de los tipos de melón evaluados. No se encontraron diferencias estadísticamente significativas entre los diferentes tipos de melón para esta variable.
Ciertamente en Costa Rica aún no se ha determinado el nivel mínimo de firmeza aceptable en frutos de melón destinados al mercado nacional, pero en el ámbito de exportación, se tiene establecido un valor mínimo recomendable de 18,6 N (J. E. MongePérez, datos sin publicar). Según este parámetro, en el presente trabajo, los melones tipo harper, galia, honey dew y charentais se ubicaron por encima de dicho valor. No obstante, en Brasil, se ha indicado que la firmeza mínima aceptable es de $22 \mathrm{~N}$ para el melón tipo galia; de $24 \mathrm{~N}$, para el melón amarillo; de $30 \mathrm{~N}$, para los melones cantaloupe y orange flesh; y de $32 \mathrm{~N}$, para el melón piel de sapo (Filgueiras, y otros, 2000). 


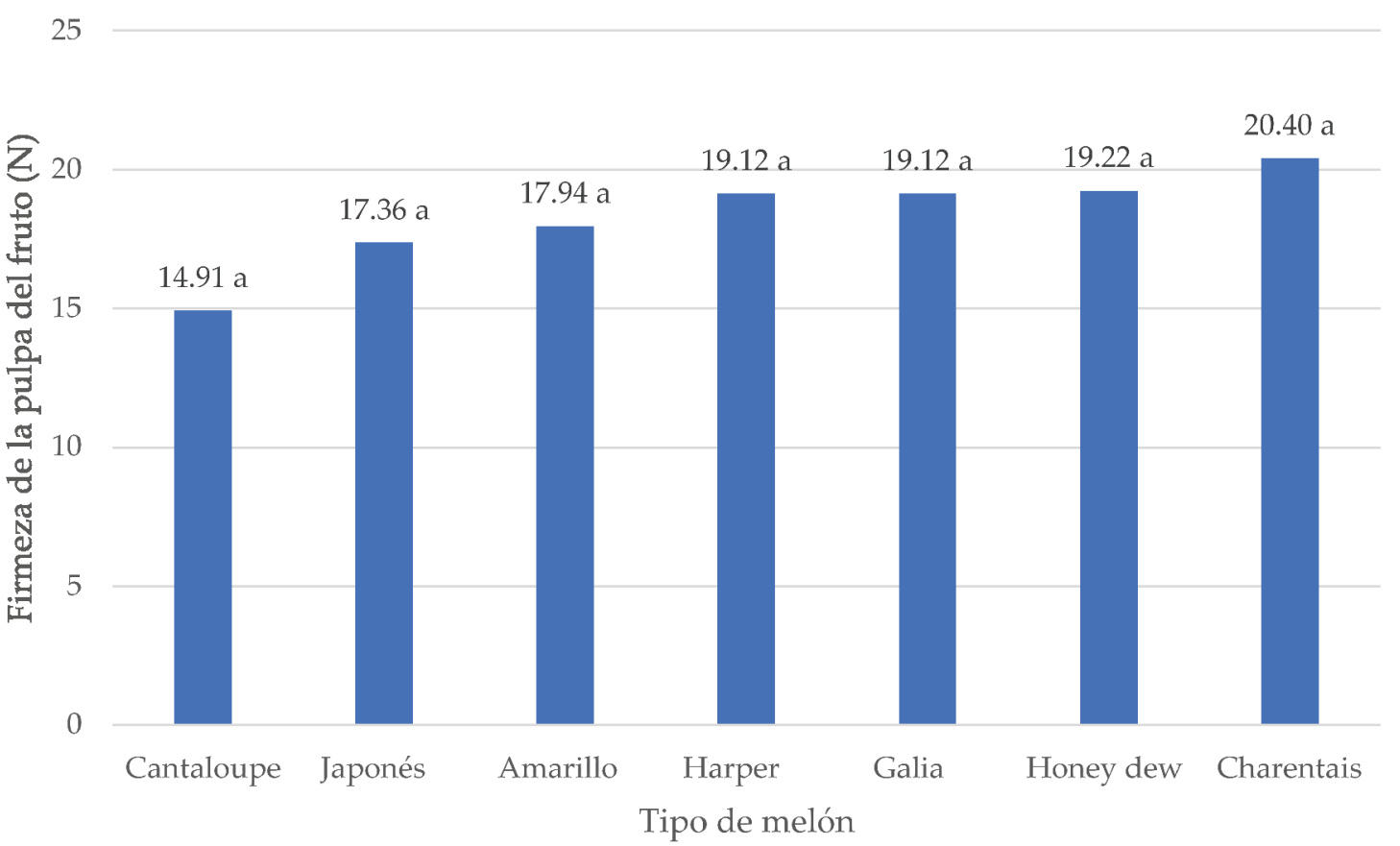

Figura 5. Firmeza de la pulpa del fruto (N), según el tipo de melón.

Nota. Elaboración propia, a partir de los datos obtenidos. Tratamientos con una letra en común no presentan diferencias significativas entre sí, según la prueba LSD Fisher $(p \leq 0,05)$.

La firmeza es un componente importante en la calidad de los frutos y es una característica intrínseca a la resistencia contra el deterioro físico y mecánico durante su acarreo y mercadeo (Gomes, Menezes, Nunes, Costa y Souza, 2001). Se considera que la firmeza de la pulpa es una característica ligada a la genética, incluso muchas veces puede ser un indicador del estado de madurez del fruto (Neibauer y Maynard, 2002).

En otras investigaciones sobre producción de melón en invernadero, se han hallado para esta variable los valores que se muestran en la tabla 7 , los cuales indican, de forma general, un rango entre 13,40 y $29,91 \mathrm{~N}$; los datos obtenidos en el presente ensayo se encuentran dentro de dicho rango. En comparación con los datos reportados por otros investigadores por tipo de melón, en el presente trabajo, se obtuvieron valores menores para los melones tipo cantaloupe $(14,91 \mathrm{~N}$, en comparación con un rango entre 25,50 y 29,91 $\mathrm{N})$; galia $(19,12 \mathrm{~N}$, en contraste con un rango entre 21,57 y $29,42 \mathrm{~N})$; honey dew $(19,22 \mathrm{~N}$, en comparación con un rango entre 24,50 y 28,40 N); y amarillo $(17,94 \mathrm{~N}$, en contraste con un reporte de $18,73 \mathrm{~N})$. Se generaron valores mayores para el melón japonés $(17,36 \mathrm{~N}$, en comparación con un rango entre 13,40 y $16,10 \mathrm{~N})$; y prácticamente el mismo valor para el melón harper $(19,12 \mathrm{~N}$, en contraste con un reporte de 19,10 N). No se encontraron datos en la literatura para el melón tipo charentais. 
Tabla 7

Firmezadelapulpadelfruto informadaen laliteraturaparaproducción demelónen invernadero, según tipodemelón

Tipo de melón Firmeza de la pulpa del fruto (N)

Referencia

\begin{tabular}{lcl}
\hline Amarillo & 18,73 & Alvarado, 2014 \\
Cantaloupe & $25,50-29,91$ & Diaz-Alvarado y Monge-Pérez, 2017 \\
Galia & $21,57-29,42$ & Abad, Garrido y Fernández, 2006 \\
Harper & 19,10 & Mitchell y otros, 2008 \\
Honey dew & $24,50-28,40$ & Díaz, 2013 \\
Japonés & $13,40-16,10$ & Charlo, Galatti, Braz y Barbosa, 2011 \\
\hline
\end{tabular}

Nota. Elaboración propia.

Porcentaje de sólidos solubles totales

En la figura 6, se presentan los datos de porcentaje de sólidos solubles totales de los tipos de melón evaluados. Los mayores valores para esta variable se obtuvieron en los melones tipo honey dew, amarillo, harper y japonés; todos con un contenido de sólidos solubles totales superior a $14^{\circ} \mathrm{Bx}$.

El contenido de sólidos solubles se utiliza como índice de clasificación de los melones de acuerdo con su grado de dulzura; los que tienen menos de $9{ }^{\circ} \mathrm{Bx}$ se consideran no comercializables, los

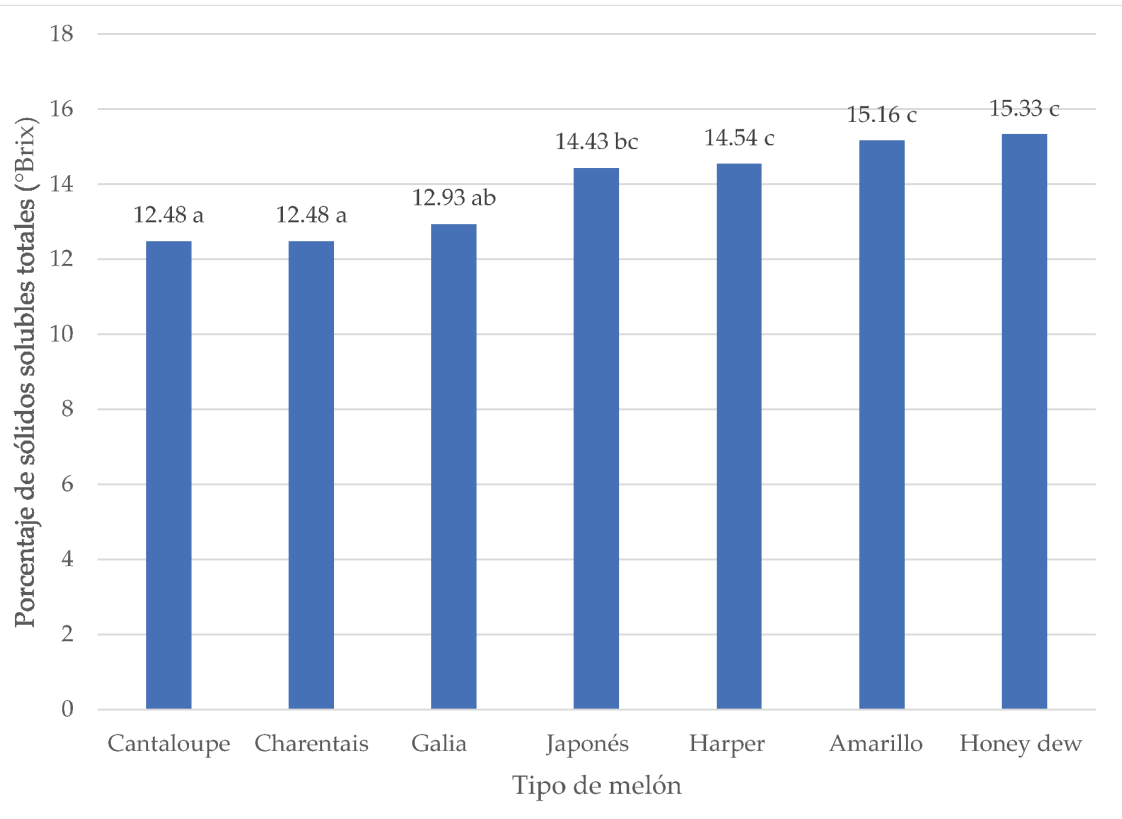

Figura 6. Porcentaje de sólidos solubles totales ( ${ }^{\circ}$ Brix), según el tipo de melón

Nota. Elaboración propia, a partir de los datos obtenidos. Tratamientos con una letra en común no presentan diferencias significativas entre sí, según la prueba LSD Fisher $(p \leq 0,05)$. 
que se encuentran entre 9 y $12{ }^{\circ} \mathrm{Bx}$ se consideran comercializables, y los que están por encima de $12{ }^{\circ} \mathrm{Bx}$ se consideran de calidad extra (Vargas et al., 2008; Rizzo y Braz, 2001). Según esos parámetros, todos los tipos de melón evaluados presentaron un porcentaje de sólidos solubles que corresponde a una calidad extra; esto indica una característica genética deseable de estos tipos de melón, así como condiciones climáticas y de manejo del cultivo idóneas para la acumulación de sólidos solubles.

En otras evaluaciones de producción de melón en invernadero, se han encontrado para esta variable los valores que se muestran en la tabla 8; de forma general, el rango se ubica entre 6,60 y $15,60{ }^{\circ} \mathrm{Bx}$, por lo que los resultados hallados en el presente trabajo se encuentran dentro de dicho rango.

Tabla 8

Porcentaje de sólidos solubles totales informado en la literatura para producción de melón en invernadero, según tipo de melón

\begin{tabular}{|c|c|c|}
\hline Tipo de melón & $\begin{array}{c}\text { Porcentaje } \\
\text { de sólidos solubles totales }\left({ }^{\circ} \text { Brix }\right)\end{array}$ & Referencia \\
\hline \multirow[t]{4}{*}{ Amarillo } & $7,50-10,90$ & Botto, 2011 \\
\hline & 10,34 & Paduan, Campos y Clemente, 2007 \\
\hline & 12,40 & $\begin{array}{l}\text { Martins, Peil, Schwengber, Assis y Mendez, } \\
1998\end{array}$ \\
\hline & 13,00 & Alvarado, 2014 \\
\hline \multirow[t]{9}{*}{ Cantaloupe } & $6,60-9,40$ & Botto, 2011 \\
\hline & $6,72-9,07$ & Morales, 2009 \\
\hline & 8,32 & Rizzo y Braz, 2001 \\
\hline & $9,30-12,70$ & Salandanan, y otros, 2009 \\
\hline & $9,40-9,80$ & Queiroga, Puiatti, Fontes y Cecon, 2008 \\
\hline & $10,55-13,05$ & Díaz-Alvarado y Monge-Pérez, 2017 \\
\hline & $11,36-11,70$ & Barrientos, 2013 \\
\hline & $11,40-12,10$ & Queiroga, Puiatti, Fontes y Cecon, 2008 \\
\hline & 83,20 & Rizzo y Braz, 2001 \\
\hline \multirow[t]{3}{*}{ Charentais } & 8,00 & Barrientos, 2013 \\
\hline & $10,20-15,10$ & Salandanan, y otros, 2009 \\
\hline & $11,20-12,40$ & Botto, 2011 \\
\hline
\end{tabular}




\begin{tabular}{lcl}
\hline Galia & Lamb, Shaw y Cantliffe, 2003 \\
$8,10-11,90$ & Shaw, Cantliffe y Taylor, 2001 \\
$8,43-9,80$ & Eltez, Tüzel y Boztok, 1999 \\
$8,70-10,10$ & Botto, 2011 \\
9,10 & Martins, Peil, Schwengber, Assis y Mendez, \\
& 1998 \\
$9,40-12,30$ & Salandanan y otros, 2009 \\
$9,90-10,50$ & Rodríguez, Shaw y Cantliffe, 2007 \\
$10,30-10,50$ & Rodríguez, Cantliffe, Shaw y Karchi, 2006 \\
$10,60-12,70$ & Abad, Garrido y Fernández, 2006 \\
11,40 & Jani y Hoxha, 2002 \\
12,30 & Paduan, Campos y Clemente, 2007 \\
$7,70-11,10$ & Botto, 2011 \\
9,50 & Barrientos, 2013 \\
11,30 & Mitchell y otros, 2008 \\
$8,20-11,70$ & Botto, 2011 \\
Harper & Díaz, 2013 \\
Honey dew & Salandanan y otros, 2009 \\
Japonés & Vargas, Castoldi, Charlo y Braz, 2008 \\
$14,20-13,50$ & Charlo, Galatti, Braz y Barbosa, 2011 \\
$9,00-11,24$ & Castoldi, Charlo, Vargas y Braz, 2008 \\
$10,35-13,05$ & Charlo, Castoldi, Vargas y Braz, 2009 \\
$10,85-13,59$ & Gualberto, Resende y Losasso, 2001 \\
$11,21-14,06$ & Rizzo y Braz, 2001 \\
$12,03-13,86$ & \\
13,15 &
\end{tabular}

Nota. Elaboración propia.

En relación con los datos informados en otras investigaciones por tipo de melón, en el presente ensayo, se obtuvieron valores mayores para los melones tipo harper $\left(14,54{ }^{\circ} \mathrm{Bx}\right.$, en comparación a un rango entre 7,70 y 11,30); japonés $(14,43$ ${ }^{\circ} \mathrm{Bx}$, en contraste con un rango entre $9,00 \mathrm{y}$ $14,06)$; amarillo $\left(15,16^{\circ} \mathrm{Bx}\right.$, en comparación con un rango entre 7,50 y 13,00$)$; y galia $\left(12,93{ }^{\circ} \mathrm{Bx}\right.$, en contraste con un rango entre 8,00 y 12,70 ). Respecto de los valores intermedios, para los melones cantaloupe $\left(12,48^{\circ} \mathrm{Bx}\right.$, en comparación con un rango entre 6,60 y 13,05); honey dew $\left(15,33^{\circ} \mathrm{Bx}\right.$, en contraste con un rango entre 8,20 y 15,60$)$; y charentais $\left(12,48^{\circ} \mathrm{Bx}\right.$ en comparación con un rango entre 8,00 y 15,10$)$. 


\section{Conclusiones y recomendaciones}

Todos los genotipos de los melones tipo amarillo, honey dew y japonés presentaron plantas andromonoicas, mientras que, en los melones cantaloupe, charentais, galia $\mathrm{y}$ harper, se presentaron genotipos monoicos $\mathrm{y}$ andromonoicos.

El mayor número de frutos por planta se obtuvo en los tipos de melón amarillo y japonés. Los frutos con mayor peso promedio se obtuvieron con los melones honey dew, cantaloupe y japonés.

El mayor rendimiento por área se obtuvo con los melones tipo japonés y honey dew $(48,89$ y 47,51 ton/ha, respectivamente); por lo tanto, se consideran los mejor adaptados a las condiciones de producción del ensayo. Este resultado se debe a que dichos tipos de melón fueron los únicos que produjeron más de 2,00 frutos por planta $\mathrm{y}$ cuyos frutos obtuvieron un peso promedio mayor a $600 \mathrm{~g}$.

No se presentaron diferencias significativas entre tratamientos para la firmeza del fruto. El mayor porcentaje de sólidos solubles totales se obtuvo con los melones tipo honey dew, amarillo, harper y japonés. Todos los tipos de melón evaluados produjeron frutos considerados de calidad extra, pues obtuvieron un porcentaje de sólidos solubles totales superior a $12{ }^{\circ} \mathrm{Bx}$.

Por una parte, se considera que el nivel de calidad y el rendimiento obtenido en las condiciones hidropónicas en que se desarrolló este ensayo es muy satisfactorio en el caso de los melones tipo japonés y honey dew, y en menor medida, en los tipos cantaloupe, harper y amarillo. Por otra parte, para los tipos galia y charentais, se deben realizar más investigaciones con el fin de lograr aumentar el rendimiento al menos hasta las 25,00 ton/ha.

Para futuras investigaciones, se recomienda evaluar diferentes tratamientos de fertilización, con el fin de intentar optimizar el nivel de producción de los diferentes tipos de melón.

\section{Agradecimientos}

Los autores agradecen la colaboración de Cristina Arguedas, María José Moya, Carolina Ramírez, Marcela Mora, Adriana Camacho, Jorge Díaz, Felipe Coto, José González, Olger Salazar, Julio Vega, Carlos González y Andrés Oviedo en el trabajo de campo; y de Mario Monge, en la revisión de la traducción del resumen al idioma inglés. Asimismo, agradecen el financiamiento recibido por parte de la Universidad de Costa Rica para la realización de este trabajo.

\section{Referencias}

Abad, D. E.; Garrido, J. C., \& Fernández, E. R. (2006). Ensayo de cultivares de melón galia (Cucumis melo L.) entutorado en invernadero. XXXV Seminario de Técnicos y Especialistas en Horticultura (241-250). Santiago de Compostela, España: Ministerio de Agricultura, Alimentación y Medio Ambiente.

Alvarado, T. S. (2014). Efecto de la aplicación de bioactivadores y del raleo manual de frutos sobre el rendimiento y la calidad de melón (Cucumis melo L. var. JMX-902) bajo cultivo protegido en La Garita, Alajuela. Santa Clara, San Carlos, Alajuela, Costa Rica: Instituto Tecnológico de Costa Rica.

Alvarado-Sánchez, T. \& Monge-Pérez, J. E. (2015). Efecto de la aplicación de bioactivadores y del raleo manual de frutos sobre el rendimiento y la calidad de melón (Cucumis melo L.) bajo cultivo protegido en Costa Rica. Tecnología en Marcha, 28(4), 15-25.

Barni, V., Barni, N. A. \& Silveira, J. R. (2003). Meloeiro em estufa: duas hastes é o melhor sistema de condução. Ciência Rural, 33(6), 1039-1043.

Barrientos, M. A. (2013). Cultivo protegido hidropónico de melón (Cucumis melo L.) en la 
zona norte de Costa Rica. Santa Clara, San Carlos, Costa Rica: Instituto Tecnológico de Costa Rica.

Bezerra, F. M.; Nunes, M. C.; Freitas, C. A. \& Silva, F. L. (2009). Desempenho de três híbridos de meloeiro sob dois espaçamentos em ambiente protegido na Chapada do Apodi. Revista Ciência Agronômica, 40(3), 412-416.

Botto, A. S. (2011). Evaluación del rendimiento y el total de sacarosa disuelta $\left({ }^{\circ} \mathrm{Bx}\right)$ de quince cultivares de melón (Cucumis melo L.) en sustrato compost y mezcla compost con arena bajo condiciones de macrotúnel. Zamorano, Honduras: Escuela Agrícola Panamericana.

Castoldi, R.; Charlo, H. C.; Vargas, P. F. \& Braz, L. T. (2008). Qualidade de frutos de cinco híbridos de melão rendilhado em função do número de frutos por planta. Revista Brasileira de Fruticultura, 30(2), 455-458.

Charlo, H. C.; Castoldi, R.; Vargas, P. F. \& Braz, L. T. (2009). Cultivo de melão rendilhado com dois e três frutos por planta. Horticultura Brasileira, 27(2), 251-255.

Charlo, H. C.; Castoldi, R.; Vargas, P. F. \& Braz, L. T. (2009). Desempenho de híbridos de melão-rendilhado cultivados em substrato. Científica, 37(1), 16-21.

Charlo, H. C.; Galatti, F. S.; Braz, L. T. \& Barbosa, J. C. (2011). Híbridos experimentais de melão rendilhado cultivados em solo e substrato. Revista Brasileira de Fruticultura, 33(1), 144-156.

Costa, C. C.; Filho, A. B.; Cavarianni, R. L. \& Barbosa, J. C. (2004). Produção de melão rendilhado em função da concentração de potássio na solução nutritiva e do número de frutos por planta. Horticultura Brasileira, 22(1), 23-27.

Díaz, J. M. (2013). Efecto de la poda y la densidad de siembra sobre el rendimiento y la calidad de fruta de melón (Cucumis melo L.) cultivado en invernadero. San Pedro de Montes de Oca, San José, Costa Rica: Universidad de Costa Rica.

Díaz-Alvarado, J. M. \& Monge-Pérez, J. E. (2017). Efecto de la poda y la densidad de siembra sobre el rendimiento y calidad de melón Cantaloupe (Cucumis melo L.) cultivado bajo invernadero. Revista Colombiana de Ciencias Hortícolas, 11(1), 21-29.

Díaz-Alvarado, J. M. \& Monge-Pérez, J. E. (2017). Producción de melón (Cucumis melo L.) en invernadero: efecto de poda y densidad de siembra. Posgrado y Sociedad, 15(1), 1-12.

Eltez, R. Z.; Tüzel, Y. \& Boztok, K. (1999). Effects of different growing media and pruning methods on greenhouse muskmelon production. Acta Horticulturae, 486, 363-367.

Filgueiras, H. A.; Menezes, J. B.; Alves, R. E.; Costa, F. V.; Pereira, L. S. \& Júnior, J. G. (2000). Colheita e manuseio pós-colheita. En R. E. Alves, Melão: pós-colheita (págs. 1-19). Brasília, Brasil: Embrapa. Recuperado de http://www.ceinfo.cnpat.embrapa.br/arquivos/artigo_1471.pdf

Gomes, J.; Menezes, J.; Nunes, G.; Costa, F. \& Souza, P. (2001). Qualidade pós-colheita de melão tipo cantaloupe, colhido em dois estádios de maturação. Horticultura Brasileira, 19(3), 356-360.

Gualberto, R.; Resende, F. V. \& Losasso, P. H. (2001). Produtividade e qualidade do melão rendilhado em ambiente protegido, em função do espaçamento e sistema de condução. Horticultura Brasileira, 19(3), 373-376.

IPGRI (2003). Descriptors for melon (Cucumis melo L.). Roma, Italia: International Plant Genetic Resources Institute.

Jani, S. \& Hoxha, S. (2002). The effect of plant pruning on production of melon grown un- 
der PVC greenhouse conditions. Acta Horticulturae, 579, 377-381.

Lamb, E. M.; Shaw, N. L. \& Cantliffe, D. J. (2003). Galia muskmelons: evaluation for Florida greenhouse production. Recuperado de http://www.hos.ufl.edu/ProtectedAg/EDIS/ HS14700.pdf

Lester, G. (1997). Melon (Cucumis melo L.) fruit nutritional quality and health functionality. Hort Technology, 7(3), 222-227.

Martins, S. R.; Peil, R. M.; Schwengber, J. E.; Assis, F. N. \& Mendez, M. E. (1998). Produção de melão em função de diferentes sistemas de condução de plantas em ambiente protegido. Horticultura Brasileira, 16(1), 24-30.

Mitchell, J. M.; Cantliffe, D. J.; Klee, H. J.; Sargent, S. A.; Stoffella, P. J. \& Tieman, D. (2008). Fruit quality and aroma characteristics of a specialty red-fleshed melon (Cucumis melo L.), 'Red Moon'. Proceedings of the Florida State Horticultural Society, 121, 274280.

Monge-Pérez, J. E. (2014). Producción y exportación de melón (Cucumis melo) en Costa Rica. Tecnología en Marcha, 27(1), 93-103.

Monge-Pérez, J. E. (2016). Evaluación preliminar de 201 genotipos de ocho diferentes hortalizas (berenjena, chile dulce, zucchini, ayote, sandía, pepino, tomate y melón) cultivados bajo invernadero en Costa Rica. En E. Solano, La investigación en Guanacaste II ( 277-300). San José, Costa Rica: Nuevas Perspectivas.

Morales, F. (2009). Caracterización de producción de genotipos de melón reticulado (Cucumis melo L.) bajo invernadero 2008-2009. Torreón, Coahuila, México: Universidad Autónoma Agraria "Antonio Narro".

Neibauer, J. \& Maynard, E. (2002). Información poscosecha:normasdecalidaddelUSDA. Recupe- rado de http://www.hort.purdue.edu/prod quality/commodities/muskmelon.html

Pádua, J. G.; Braz, L. T.; Banzatto, D. A.; Gusmão, M. T. \& Gusmão, S. A. (2003). Net melon cultivars productivity under different cultivation systems, during summer and winter. Acta Horticulturae, 607, 83-89.

Paduan, M. T.; Campos, R. P. \& Clemente, E. (2007).Qualidadedosfrutos detipos demelão, produzidos em ambiente protegido. Revista Brasileira de Fruticultura, 29(3), 535-539.

Purquerio, L. F.; Filho, A. B. \& Barbosa, J. C. (2003). Efeito da concentração de nitrogênio na solução nutritiva e do número de frutos por planta sobre a produção do meloeiro. Horticultura Brasileira, 21(2), 186-191.

Queiroga, R. C.; Puiatti, M.; Fontes, P. C. \& Cecon, P. R. (2008). Partição de assimilados e índices fisiológicos de cultivares de melão do grupo Cantalupensis influenciados por número e posição de frutos na planta, em ambiente protegido. Revista Ceres, 55(6), 596-604.

Queiroga, R. C.; Puiatti, M.; Fontes, P. C. \& Cecon, P. R. (2008). Produtividade e qualidade de frutos de meloeiro variando número de frutos e de folhas por planta. Horticultura Brasileira, 26(2), 209-215.

Queiroga, R. C.; Puiatti, M.; Fontes, P. C. \& Cecon, P. R. (2008). Produtividade e qualidade do melão cantaloupe, cultivado em ambiente protegido, variando o número e a posição dos frutos na planta. Bragantia, 67(4), 911-920.

Rizzo, A. A. \& Braz, L. T. (2001). Características de cultivares de melão rendilhado cultivadas em casa de vegetação. Horticultura Brasileira, 19(3), 237-240.

Rodríguez, J. C.; Cantliffe, D. J.; Shaw, N. L. \& Karchi, Z. (2006). Soilless media and contai- 
nersforgreenhouse production of 'Galia' type muskmelon. Hort Science, 41(5), 1200-1205.

Rodríguez, J. C.; Shaw, N. L. \& Cantliffe, D. J. (2007). Influence of plant density on yield and fruit quality of greenhouse-grown Galia muskmelons. Hort Technology, 17(4), 580-585.

Salandanan, K.; Bunning, M.; Stonaker, F.; Külen, O.; Kendall, P. \& Stushnoff, C. (2009). Comparative analysis of antioxidant properties and fruit quality attributes of organically and conventionally grown melons (Cucumis melo L.). Hort Science, 44(7), 1825-1832.
Shaw, N. L.; Cantliffe, D. J. \& Taylor, B. S. (2001). Hydroponically produced 'Galia' muskmelon - what's the secret? Proceedings of the Florida State Horticultural Society, 114, 288-293.

Torres, J. M. (1997). Los tipos de melón comerciales. En A. Namesny, Melones (13-20). España: Ediciones de Horticultura.

Vargas, P. F.; Castoldi, R.; Charlo, H. C. \& Braz, L. T. (2008). Qualidade de melão rendilhado (Cucumis melo L.) em função do sistema de cultivo. Ciência e Agrotecnologia, 32(1), 137-142. 